\title{
Characterization of a copper spark discharge plasma in argon atmosphere used for nanoparticle generation
}

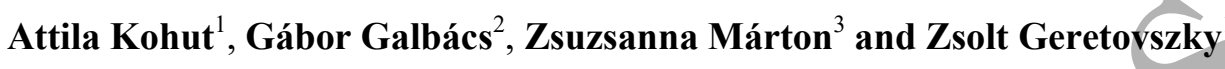 \\ ${ }^{1}$ Department of Optics and Quantum Electronics, University of Szeged, Szeged H-6720, \\ Dóm tér 9, Hungary, \\ ${ }^{2}$ Department of Inorganic and Analytical Chemistry, University of Szeged, Szeged H-6720, \\ Dóm tér 7, Hungary \\ ${ }^{3}$ MTA-PTE High-field Terahertz Research Group, University of Pécs, Pécs H-7624, \\ Ifjúság útja 6, Hungary
}

E-mail: akohut@titan.physx.u-szeged.hu

\begin{abstract}
Spark discharge nanoparticle generation is a dynamically developing application of discharge plasmas. In the present study a spark plasma used for nanoparticle generation is characterized by means of spatially and temporally resolved optical emission spectroscopy (OES) supplemented by fast imaging. The data acquired during the generation of copper nanoparticles in argon ambient is used to describe the spatial and temporal evolution of the species in the spark gap and to derive plasma parameters such as excitation temperature and electron concentration on one hand, and the concentration of the $\mathrm{Cu}$ species eroded by a single spark on the other. It is shown that temporally and spatially resolved OES together with a simple equilibrium model are efficient tools to estimate the characteristics of the spark discharge plasma that typically exists in spark discharge nanoparticle generators.
\end{abstract}

Keywords: Spark discharge, nanoparticle generation, plasma diagnostics, optical emission spectroscopy, copper, argon, multi-element Saha-Boltzmann plot

\section{Introduction}

Spark discharge sources are widely used in diverse areas of science and technology. One of the most dynamically developing fields of application is the generation of nanoparticles (NPs). After the introduction of the so-called spark discharge generators (SDGs) [1] the technique established itself as a distinguished physical method of NP production [2-4]. SDGs employ high-voltage and high-current microsecond-long oscillatory discharges, initiated in a controlled gas environment, in order to erode a pair of electrodes serving as a primary source of the desired NPs. This provides a versatile, clean and efficient method of NP production [2,5] that also has a strong potential for up-scaling $[4,6]$, that was convincingly demonstrated in the BUONAPART-E project [7] funded by the European Union's $7^{\text {th }}$ Framework Program. 
Several aspects of NP generation in spark discharges have been investigated in the literature. Most of these studies focused on the physical and chemical characterization of the produced particles [5, 8-13] and potential particle formation mechanisms [14-16], but only a couple of attempts have been made to describe the characteristics of the plasma in the SDG during NP generation (e.g. [17, 18]). However, the fundamental description of the SDG spark is clearly a very important step towards the understanding of elementary processes leading to NP formation and hence towards gaining better control over NP production.

The literature of other spark plasma sources can serve as an important input to the investigation of the spark discharge specific to NP generators. The wide range of applications of electrical spark discharges produced numerous studies aiming for describing the properties of the spark and correlating them with the (physical) processes taking place in the discharge gap (e.g. [19-22]). For example, great efforts were made as early as in the middle of the $20^{\text {th }}$ century towards the description of processes taking place in analytical spark discharges (e.g. [23-26]). The following four stages are generally distinguished during the evolution of a high-voltage spark discharge: i) pre-breakdown, ii) breakdown, iii) arc, and iv) afterglow [26, 27]. The temporal starting point of this classification is the moment of application of the high voltage to the gap.

During the pre-breakdown stage, the electrons emitted from the cathode or formed in the gas due to the ionizing environmental radiation gain sufficient energy to produce collisional ionization. The result is an electron avalanche and the eventual "breaking down" of the gap. If the electric field of the space charge of the primary electron avalanche is sufficiently high, a thin conducting channel (streamer) is formed, which bridges the electrodes [28]. Depending on the size of the electrode gap, the gas atmosphere, electrode configuration and other discharge parameters the breakdown can be also driven by the Townsend mechanism of avalanche multiplication, and not by streamers [28]. The prebreakdown stage ends when a conducting channel is formed between the electrodes and the spark enters the breakdown stage. In SDGs, the formation of this conducting channel takes place in an unregulated fashion, i.e. when the increasing voltage, applied to the electrode pair, reaches the breakdown voltage of the gas in the gap. Since in SDGs the total inductance of the discharge loop is small, the duration of the breakdown stage is short, typically last a few tens of ns [27].Channel formation is accompanied by an intense light emission from multiply charged ionic lines of the gas. The discharge channel allows for a high current to flow in the discharge loop which will eventually lead to the erosion of the electrodes.

The discharge enters the so-called arc stage when the erosion of the electrodes, that is melting and evaporation of the electrode material, as well as ejection of small particles, starts. This stage is usually reached in less than $1 \mu \mathrm{s}$ [29]. As a consequence of the erosion process, the gap will no longer contain only the atomic and ionic species of the ambient gas, but also the species of the electrode material. The arc stage, which is accompanied by concomitant light emission of the electrode and gas species, lasts until the electrical current ceases. This lasts a few or a few tens of microseconds, depending on the electrical parameters of the discharge loop. The emission characteristics of the arc stage of an analytical spark was investigated in great detail (see e.g. [23]) and peak plasma temperatures as high as 30000-40000 K have been reported [30, 31], together with electron concentration in the range of $10^{17} \mathrm{~cm}^{-3}$ [31]. However, most of the works focusing on plasma properties (instead of the emission characteristics itself) were obtained on unipolar discharges in which the oscillatory nature of the spark is suppressed, which is beneficial for analytical purposes. Bye and Scheeline have investigated the properties of analytical spark plasmas in a series of papers [32-34]. They reported an average electron temperature of about $16500 \pm 450 \mathrm{~K}$ for an analytical spark ignited between $\mathrm{Cu}$ and $\mathrm{W}$ electrodes in $\mathrm{Ar}$ and peak electron concentration of $2.5 \pm 0.3 \times 10^{17} \mathrm{~cm}^{-3}$ [32].

It is a general experimental observation that light emission from the gap does not cease when the electrical current terminates, but the emission from excited atoms can still be observed for a relatively 
long time afterwards, i.e. in the so-called afterglow stage [26]. The duration of the afterglow is much longer than that of the arc stage, e.g. tens or even hundreds of microseconds long, as opposed to a few microseconds. However, since the afterglow has much less relevance from a spectroanalytical point of view, this stage has been investigated in less detail in the literature as compared to the arc stage. It is our opinion though that from the point of view of NP formation the afterglow certainly deserves more attention, as the presence of afterglow actually indicates that excited atomic species from the electrode material are still present in the gap for an extended time and the amount of material released can also serve as an input to the NP formation, when relaxed.

The aim of the present study is to characterize the SDG spark plasma during the arc and the afterglow/ stages by means of spatially and temporally resolved optical emission spectroscopy (OES) supplemented by fast imaging. The data acquired during the generation of $\mathrm{Cu}$ NPs in Ar ambient is used to describe the spatial and temporal evolution of the species in the spark gap and to derive plasma parameters such as excitation temperature, electron concentration, and the concentration of plasma species. The results obtained are intended to support the understanding of NP formation process taking place in the SDG and to provide experimental data for the validation of NP generation models.

\section{Methods}

\subsection{The spark discharge generator}

The schematic top view of the experimental setup is shown in figure 1. The chamber geometry was optimized for NP production [7], and the chamber itself/was manufactured by Pfeiffer Vacuum $\mathrm{GmbH}$. It is a KF-sealed, DN-160 sized, cylindrical stainless steel chamber with four, radially oriented KF-40 ports. The chamber was set up in an upright position, in which the two large KF-160 ports were located on the sides. One of the KF-160 ports was sealed by a blank flange, whereas the other was equipped with a fused silica window in order to facilitate optical observation.

The $\mathrm{Cu}$ electrode pair (99.9\% purity, Kurt J. Lesker Co.)/was horizontally positioned and axially aligned. The inter-electrode distance (i.e. electrode gap) was controlled by micropositioners (Model K150-BLM-1, MDC Vacuum Ltd.) and set to $2.0 \mathrm{~mm}$ for all results reported herein. Cylindrical copper electrodes of $3.00 \mathrm{~mm}$ diameter with an apex angle of 30 degree were used which were preeroded to a radius of curvature of $10 \mathrm{~mm}$. The effect of erosion on the inter-electrode distance was balanced by re-adjusting the electrodes and keeping the breakdown voltage constant.

A $99.996 \%$ purity argon (Messer Hungarogáz Ltd.) carrier gas flow, entering the chamber via the top KF-40 port (downward pointing "crossflow") was employed during NP generation. The gas flow rate was controlled by a mass flow controller (Model GFC16, Aalborg Instruments \& Controls, Inc.) and set to $5 \mathrm{slm}$. All experiments were carried out at atmospheric pressure, maintained by a diaphragm pump and monitored by a piezo-resistive pressure gauge (Model VD81, Pfeiffer Vacuum $\mathrm{GmbH} /$ Thyracont Vacuum Instruments $\mathrm{GmbH}$ ).

A simple capacitor charging circuit fed the spark discharge. A monolithic, high voltage, pulse discharge capacitor (Model 450PM980, General Atomics) with a capacitance of $8 \mathrm{nF}$ was connected parallel to the spark gap and charged by a high voltage capacitor charging power supply (Model HCK 800-12500, FuG GmbH). The discharge of the capacitor between the electrodes commences when the voltage on the capacitor reaches the breakdown voltage of the gas in the electrode gap. The resulting spark discharge is a bipolar, oscillatory discharge, the damping coefficient and the oscillation frequency of which are set by the net impedance (i.e. the total capacitance, resistance and inductance) of the discharge loop. The repetition rate of the discharges (spark repetition rate, SRR) was kept constant at $100 \mathrm{~Hz}$ during the measurements. The duration of the oscillatory discharge (the characteristic duration of the exponentially decaying current waveform) in the present experiments was about $4 \mu$ s with peak currents of about $250 \mathrm{~A}$. 


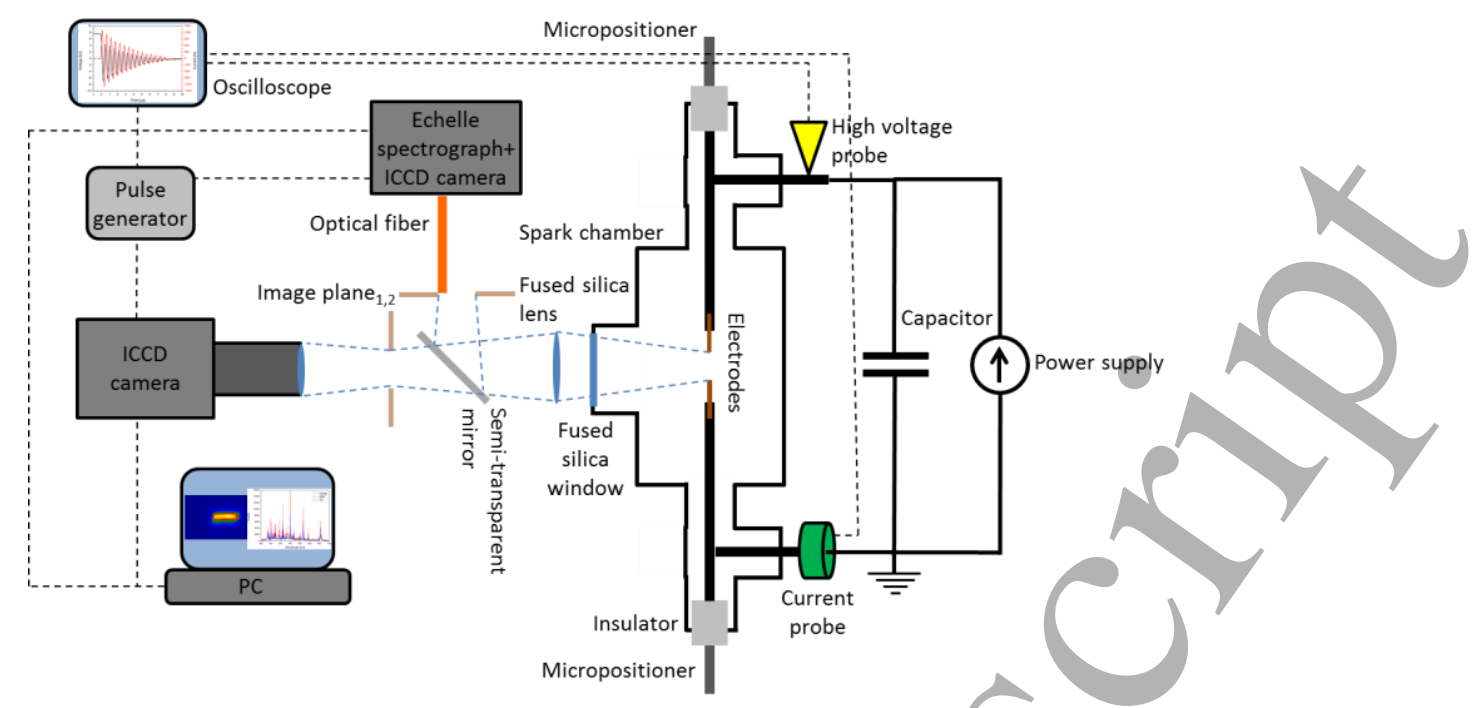

Figure 1. Schematic top view of the experimental setup. For the sake of clarity, only the used ports of the chamber are shown. Please note that the electrodes' axis was actually horizontal, with the gas flowing downwards from the top (cross flow).

\subsection{Electrical and optical measurements}

The voltage and current waveforms in the discharge loop were recorded by a $200 \mathrm{MHz}$ digital storage oscilloscope (Model DSOX2024A, Keysight Technologies Inc.) using a broad-band high voltage probe (Model TT-HVP 15HF, Testec Elektronik GmbH.) and a current probe (Model 110, Pearson Electronics, Inc.), respectively.

Two 1:1 images of the emitting spark plasma were produced by using a fused silica lens $(9 \mathrm{~cm}$ focal length) and a semi-transparent mirror (ND10A, Thorlabs $\mathrm{GmbH}$ ), as can be seen in figure 1. One of the images was used to facilitate temporally and spatially resolved optical emission spectroscopy (OES) measurements, whereas the other was used to record time-resolved images of the spark gap and its vicinity. Spatial resolution of the OES experiments was provided by positioning the bare end of a multimode, silica optical fibre with a $50 \mu \mathrm{m}$ core and 0.22 numerical aperture (SFS50/125Y, Thorlabs $\mathrm{GmbH}$ ) to different locations within the image. The other end of the fibre was connected to an Echelle spectrograph (Model Mechelle 5000, Andor Technology Ltd.) equipped with a high sensitivity, nsgated intensified CCD (ICCD) detector (Model iStar-734 Gen 2 (W-AGT, -03), Andor Technology Ltd.). This setup allowed for the collection of spectra in the $300-800 \mathrm{~nm}$ range and provided spectral, temporal, and spatial resolutions of ca. $0.15 \mathrm{~nm}, 500 \mathrm{~ns}$, and $50 \mu \mathrm{m}$, respectively. The spectral sensitivity of the optical system was corrected by using a NIST-traceable deuterium-halogen calibration lamp (Model DH-2000-CAL, Ocean Optics, Inc.). The correction was checked before each experimental run and repeated if necessary. The imaging data was collected in parallel with the OES measurements using a separate ICCD camera (Model iStar DH334T-18F-04, Andor Technology Ltd.) equipped with a UV camera lens (Model UV1054B, Kogaku Co. Ltd.) having a temporal and spatial resolution of $100 \mathrm{~ns}$ and ca. $10 \mu \mathrm{m}$, respectively. The ICCD cameras were triggered from the rising edge of the current signal of the current probe, which ensured reproducible triggering at a fixed threshold current. The minimum data collection delay was $70 \mathrm{~ns}$ which were taken into account during the interpretation of experimental results.

The temporal and spatial characteristics of the emission from each species studied were confirmed by measurements done at several wavelengths, but for the sake of clarity, we only show data for one selected spectral line per species below. 


\subsection{Methods used for the evaluation of plasma diagnostic data}

The OES data were used to derive the temperature and the electron concentration of the spark plasma. The Boltzmann plot method (see e.g. $[35,36]$ ) is a widely used method for calculating the so-called excitation temperature of the plasma. Here we have to assume, that the system is in local thermodynamic equilibrium (LTE), therefore the population of the excited states obey the Boltzmann distribution. As a consequence, the emitted intensity of a transition between two energy levels $(i \rightarrow j)$ can be written as:

$$
I_{i j}=h v_{i j} A_{i j} \frac{1}{Z} g_{i} n e^{\left(\frac{-E_{i}}{k T}\right)}
$$

where $A_{i j}$ is the transition probability, $Z$ is the partition function, $g_{i}$ and $E_{i}$ are the statistical weight and the energy of the excited level, respectively, $k$ and $h$ is the Boltzmann and Planck's constant, respectively, $T$ is the excitation temperature, $v_{i j}$ is the frequency of the transition, and $n$ is the number concentration of the studied species. If we rearrange (1) it is apparent that different spectral lines of a selected species scatter around a straight line when $\ln \left(\frac{I_{i j}}{v_{i j} g_{i} A_{i j}}\right)$ is plotted as a function of $E_{i}$ :

$$
\ln \left(\frac{I_{i j}}{v_{i j} g_{i} A_{i j}}\right)=\ln \left(\frac{h n}{Z}\right)-\frac{E_{i}}{k_{B} T}
$$

The slope of the line as defined by (2) can be used to derive the excitation temperature of the given species. This form of the method can be used only for a single species at a given ionization level. However, (2) can be extended to a given species at different ionization levels or to different species at the same ionization level if additional information on the plasma is known. The former is called the Saha-Boltzmann method (see e.g. $[32,36]$ ) while the latter is the so called multi-element SahaBoltzmann method [36].

When LTE exists in the plasma it is assumed that at a given spatial position all species have the same temperature which, together with the electron concentration, determines the concentration of the species at different ionization levels through the Saha equation:

$$
n_{+}=\frac{1}{n_{e}} 2 \frac{Z_{+}}{Z}\left(\frac{2 \pi m_{e} k_{B} T}{h^{2}}\right)^{\frac{3}{2}} \exp \left(-\frac{W_{+}-\Delta W}{k_{B} T}\right) n \equiv S n,
$$

where $n_{e}$ is the number concentration of electrons, $W_{+}$is the ionization energy of the studied species and $\Delta W$ is the lowering of the ionization energy [40]. The + subscript refers to ions. By combining (2) and (3) the following generalized equation can be written for a given species at different ionization levels:

$$
\ln \left(\frac{I_{i j} Z}{h v_{i j} g_{i} A_{i j} S}\right)=\ln (n)-\frac{E_{i}}{k_{B} T^{\prime}}
$$

where $S$ is unity for atoms and can be calculated for each ionization levels as a function of the temperature and the electron concentration. Plotting ions and atoms on the same graph obviously widens the energy spread of the species and hence increases the accuracy of the temperature determination. The validity of LTE assumption can also be inferred from the linearity of the plot. However, (4) requires the knowledge of the electron concentration and $Z$ and $S$ also contain the temperature, hence it usually requires an iterative process for determining the temperature.

The Saha-Boltzmann method was applied to selected atomic and ionic Ar lines of the emission spectrum of the spark plasma in the arc stage, while the multi-element method (to be discussed later) was employed on atomic $\mathrm{Cu}$ and $\mathrm{Ar}$ lines during the afterglow. In all cases, well resolved spectral lines were selected for the calculations which do not interfere with adjacent spectral lines. The tabulated data of selected transitions were taken from the NIST database [37] and are listed in table 1. 
Table 1. Atomic data of selected $\mathrm{Ar}$ and $\mathrm{Cu}$ transitions; $\lambda_{i j}$ is the emission wavelength, $E_{i}$ and $g_{i}$ are the energy and the statistical weight of the upper level and $A_{i j}$ is the transition probability of selected atomic transitions, respectively.

\begin{tabular}{|c|c|c|c|c|c|}
\hline Spectrum & $\lambda_{i j}[\mathrm{~nm}]$ & $E_{i}\left[\mathrm{~cm}^{-1}\right]$ & $g_{i}$ & $A_{i j}\left[\times 10^{7} \mathrm{~s}^{-1}\right]$ & $A_{i j}$ accuracy [\%] \\
\hline Ar I & 738.40 & 107290 & 5 & 0.85 & $\leq 25$ \\
\hline $\operatorname{Ar} I$ & 763.51 & 106237 & 5 & 2.45 & $\leq 25$ \\
\hline $\operatorname{Ar} I$ & 696.54 & 107496 & 3 & 0.64 & $\leq 25$ \\
\hline Ar I & 794.82 & 107132 & 3 & 1.86 & $\leq 25$ \\
\hline Ar II & 448.18 & 173393 & 6 & 4.55 & $\leq 10$ \\
\hline Ar II & 457.93 & 161089 & 2 & 8.00 & $\leq 10$ \\
\hline Ar II & 458.99 & 170401 & 6 & 8.20 & $\leq 3$ \\
\hline Ar II & 480.60 & 155043 & 6 & 7.90 & $\leq 3$ \\
\hline Ar II & 484.78 & 155708 & 2 & 8.50 & \\
\hline Ar II & 487.99 & 158730 & 6 & 7.80 & $\leq 3$ \\
\hline Ar II & 496.51 & 159393 & 4 & 3.94 & $\leq 3$ \\
\hline Ar II & 506.20 & 155351 & 4 & 2.23 & $\leq 10$ \\
\hline Ar II & 465.79 & 159706 & 2 & 8.10 & $\leq 10$ \\
\hline Ar II & 476.49 & 160239 & 4 & 5.75 & $\leq 10$ \\
\hline Ar III & 328.58 & 204803 & 7 & 20 & $\leq 50$ \\
\hline Ar III & 330.19 & 204656 & 5 & 20 & $\leq 50$ \\
\hline Ar III & 331.12 & 204570 & 3 & 20 & $\leq 50$ \\
\hline $\mathrm{Cu} \mathrm{I}$ & 521.82 & 49942 & 6 & 7.50 & $\leq 18$ \\
\hline $\mathrm{Cu} \mathrm{I}$ & 465.11 & 62403 & 8 & 3.80 & $\leq 18$ \\
\hline $\mathrm{Cu} \mathrm{I}$ & 510.55 & 30783 & & 0.20 & $\leq 18$ \\
\hline $\mathrm{Cu} \mathrm{I}$ & 515.32 & 49935 & & 6.00 & $\leq 18$ \\
\hline $\mathrm{Cu} \mathrm{I}$ & 578.21 & 30535 & & 0.17 & $\leq 18$ \\
\hline $\mathrm{Cu} \mathrm{I}$ & 458.70 & 62948 & 6 & 3.20 & $\leq 18$ \\
\hline
\end{tabular}

The effect of self-absorption, which could potentially bias the results of the temperature calculation, was tested by using the double light path method [38] and was found to be negligible at the spectral lines used. It should be noted, that the accuracy of the excitation temperature values obtained can be significantly influenced by the accuracy of the transition probabilities, which is also tabulated in table 1.

During the arc stage the electron concentration in the spark channel was calculated from the Stark broadening of the emitted spectral lines. The Stark effect dominantly depends on the electron impacts while the electric microfields of ions have a minor contribution. In the present study the broadening of the $460.96 \mathrm{~nm}$ Ar II line was used to derive the electron concentration. In case of ionic lines, the contribution of ions to the Stark broadening is negligible and the Stark width, according to Konjevic [38], can be approximated by:

$$
w\left(N_{e}, T_{e}\right)=w_{e}\left(T_{e}\right) \cdot N_{e} \cdot 10^{-17} \mathrm{~cm}^{3}
$$

where $w$ is the full width at half maximum (FWHM) of the Lorentzian line profile associated to the Stark broadening, $N_{e}$ is the electron concentration and $w_{e}$ is the electron impact FWHM at $N_{e}$ $=10^{17} \mathrm{~cm}^{-3}$ and $T_{e}$ is the electron temperature. As can be seen in (5), the electron concentration can be derived from the Stark width of a spectral line if $w_{e}$ is known at the given electron temperature. However, before applying (5) to a measured line profile other broadening mechanisms, such as natural, Doppler, collisional and instrumental broadening have to be considered. We determined, by using a low-pressure calibration source, that in our case the most dominant mechanism is instrumental 
broadening, which causes a Gaussian line profile (which has an FWHM of $0.128 \mathrm{~nm}$ in the present case). The Stark width of the spectral line was therefore obtained by fitting the measured line profile with a Voigt profile, having a Gaussian component of fixed width describing the instrumental broadening. It should be noted that the derived electron concentrations have uncertainties around $25 \%$, limited by the accuracy of the electron impact width values used in (5). The electron width values used here were taken from [39]. The OES data processing and calculations were carried out by using Origin (Originlab Corporation) and our purpose-made scripts written in Python 3.5 programming language and MATLAB (MathWorks, Inc.).

\section{Results and discussion}

For NP generation the arc and afterglow stages of the spark discharge are of interest. These two stages have substantially different contributions to the production of atomic vapours that ultimately leads to NP formation in the gas phase. During the arc stage, the conducting channel is fed by the electrical energy of the capacitor (and hence alternating current flows in the discharge loop), while in the afterglow stage the discharge receives no further external (electrical) energy input. The characteristics of these two domains will be individually discussed in detail in the following chapters.

\subsection{Characterization of the discharge in the arc stage}

The discharge enters the arc stage after the breakdown is completed and hence current is able to flow through the conducting plasma between the electrodes. In the present experiments the breakdown completes in 40-50 ns, as indicated by the displacement current waveform derived from the measured voltage signal. As a consequence, in our setup it is ensured that the spark discharge is in the arc stage even at the minimum delay of the spectral acquisition (see the Methods sections for details). As it was pointed out in the Introduction, the arc stage of the spark is accompanied by intense light emission, which is dominated by the spectral lines of ions and atoms of the ambient gas. The temporal behaviour of the intensity of the emission from several ionic species measured in the centre point of the spark gap is shown in figure 2(a). The light emission of $\mathrm{Ar}^{+}$and $\mathrm{Ar}^{2+}$ follows the current oscillations in the discharge loop and is only detectable while electric current flows between the electrodes. This is due to the fact that the ionization (and excitation) energies of these species are high thus can only be supplied by the discharge when the temperature and electron concentration is the highest [26]. This implies that argon ions emit in the close proximity of the current-conducting channel bridging the electrodes, therefore the light emission of these ions can be used to monitor the properties of the current-carrying channel. Apart from the emission of the ionic species, atomic argon emission is also detectable from the very beginning of the arc stage as evidenced by the temporally resolved spectra (see Figure 4(a)). This could mean that the current-carrying channel is surrounded by a colder region containing argon atoms which are the source of this atomic emission. However, as it will be shown later, it is more likely that argon atoms and ions coexist in the conducting spark channel and have the same temperature. This does not mean though that argon atoms could not spread farther apart from the electrodes' axis as time passes (cf. 2(b) and Figure 4(b)).

Unfortunately $\mathrm{Cu}^{+}$could only be detected with a very low signal-to-noise ratio, which is probably due to the reduced sensitivity of our instrumentation in the UV range, where dominant $\mathrm{Cu}$ II lines emit. The approximate peak time of the light emission from $\mathrm{Cu}^{+}$differs significantly from that of the argon species. This is because copper species are much less abundant in the gap at early times, since they are only produced in the arc stage. 

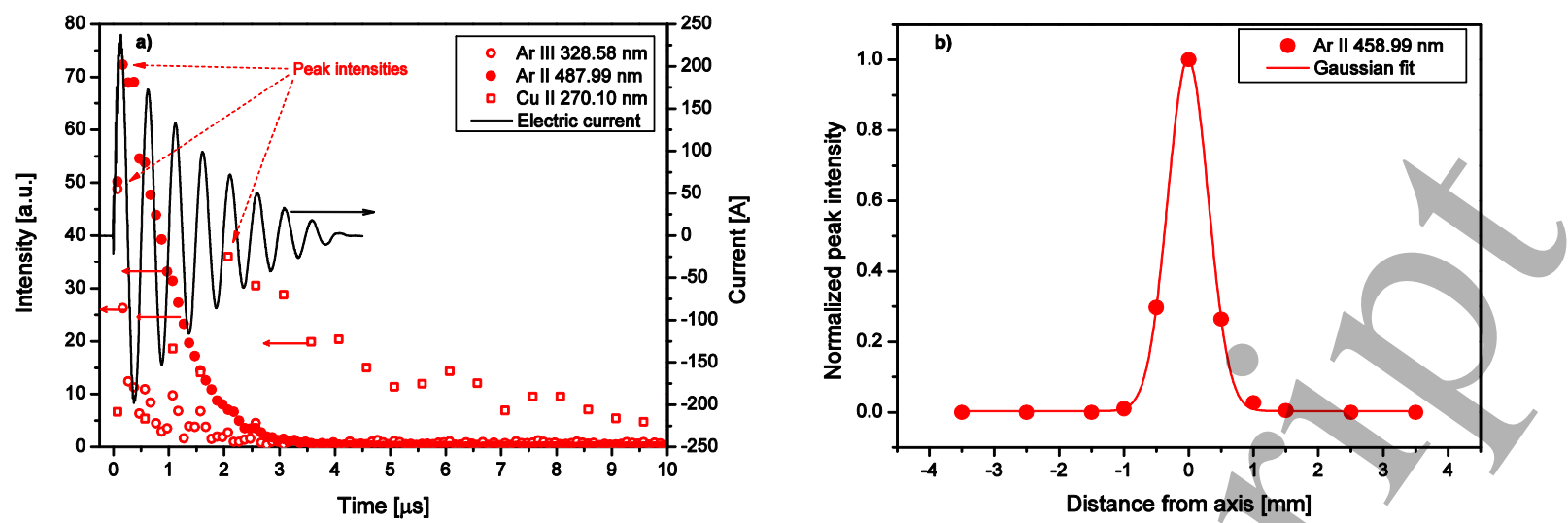

Figure 2. Time evolution of the intensity of some selected ionic spectral lines, emitted from the centre of the gap (a). The spatial variation of the peak intensity of an ionic Ar spectral line perpendicular to the electrodes' axis, at the centre of the gap (b).

In order to reveal the spatial extent of the spark channel, as defined by the Arions during the arc stage, a $7 \mathrm{~mm}$ wide area perpendicular to the direction of the common axis of the electrodes was scanned at the centre of the gap (i.e. in the direction of the argon flow). Time evolution curves, similar to those shown in figure 2(a), were constructed from the recorded emission spectra. In figure 2(b), the emission intensity at the maximum of the time evolution curves (denoted as peak intensities on figure 2(a)) of the Ar II line is plotted as a function of spatial position. Within the time-resolution of the present experiments the emission lines of $\mathrm{Ar}^{+}$ions reach their peak intensity simultaneously across a $2 \mathrm{~mm}$ wide area at around $500 \mathrm{~ns}$ after the onset of the breakdown. As can be seen in figure 2(b), the spatial distribution of the emission intensity of the Ar II species has a full width at half maximum (FWHM) of about $0.7 \mathrm{~mm}$. This represents the diameter of the spark channel when the intensity of the given spectral line is at maximum, that is, when the formation of the channel is finished. It should be noted, that the spark channel evolves in time, which results in the broadening of the channel. An approx. threefold increase in diameter was recently reported by the end of the arc stage, without spectral resolution, in an SDG operating in nitrogen atmosphere [18].

The electron concentration was derived from the Stark broadening of Ar II lines as described in the Methods section. The temporal evolution of the electron concentration in the spark channel (reported here for the centre of the spark gap) is shown in figure 3. As can be seen its value decreases from $\sim 7 \times 10^{17} \mathrm{~cm}^{-3}$ to $\sim 2 \times 10^{17} \mathrm{~cm}^{-3}$ as the amplitude of the oscillating current decays. The three datasets plotted in figure 3 also prove, that the electron concentration is very similar across the $0.7 \mathrm{~mm}$ diameter plasma channel.

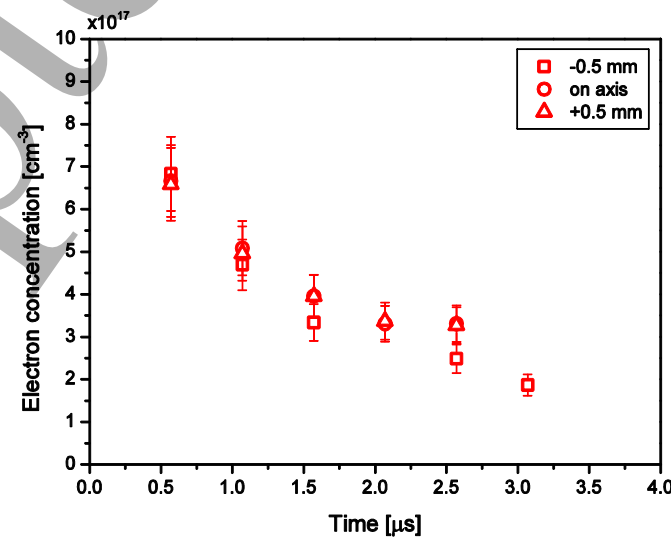

Figure 3. The electron concentration of the spark plasma during the arc stage at different spatial locations (on axis and 2 radially symmetric positions). 
In accordance with the literature (e.g. [32, 41]) we assume that the LTE condition holds in the arc stage. Based on this assumption, the excitation temperature can be derived from the intensity of selected spectral lines by using the Boltzmann plot method (see the Methods section for further details). The existence of LTE could be checked by comparing the temperature values derived from the spectra of different species (see e.g. [32]). Unfortunately in the present case the energy spread of the apparent spectral lines allowed for temperature determination only for $\mathrm{Ar}^{+}$ions. However, as it was pointed out in the Methods section, indirect evidence on the existence of LTE can be found by constructing the Saha-Boltzmann plot of argon species at different ionization levels, namely $\mathrm{Ar}^{0}, \mathrm{Ar}^{+}$ and $\mathrm{Ar}^{2+}$. Figure 4(a) shows the Saha-Boltzmann plots obtained at different delays in the spark gap. Both Ar III, Ar II and Ar I lines are incorporated in the graph where the quality of the experimental data allowed us to do so.
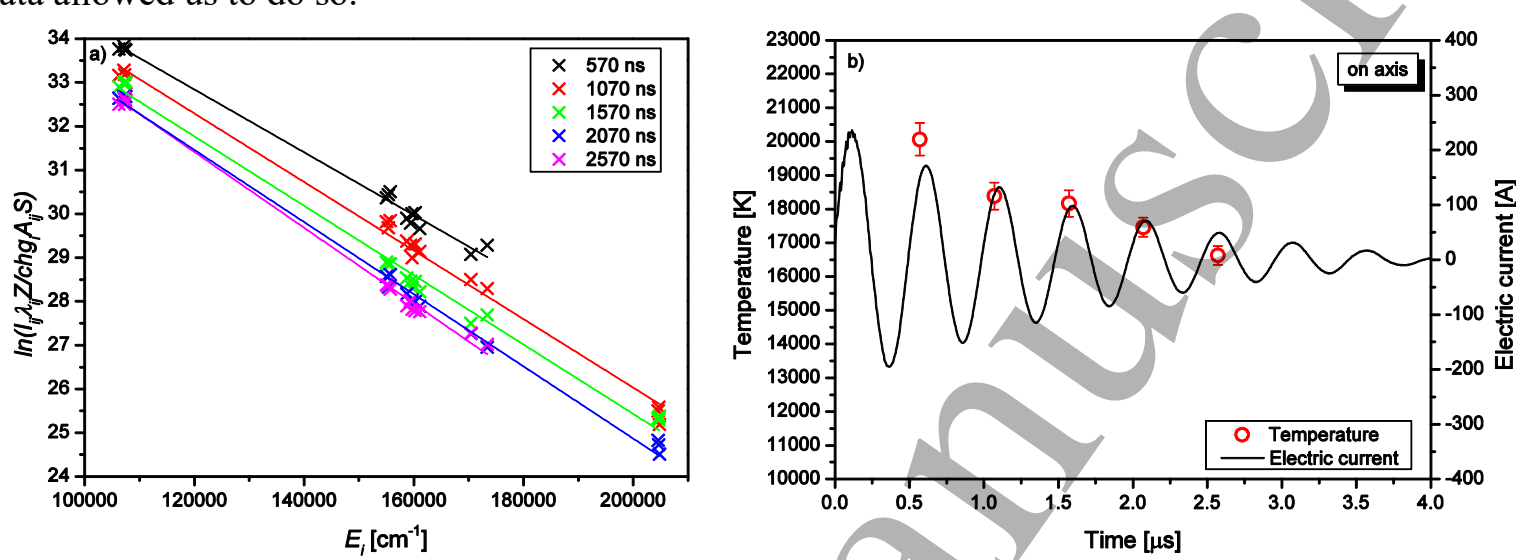

Figure 4. Saha-Boltzmann plots obtained in the spark gap at different time delays (a), and the variation of the temperature together with the oscillating electric current (b). The temperature values were derived from the acquired emission spectra which were taken with a gate of $500 \mathrm{~ns}$ at every $500 \mathrm{~ns}$ starting from about $70 \mathrm{~ns}$ after the rising of the first current peak.

As it can be seen in figure 4(a), the Saha-Boltzmann plots of argon atoms and ions show small scatter and good linearity up until 2500 ns delays. This provides a strong indication that $\mathrm{Ar}^{0}, \mathrm{Ar}^{+}$and $\mathrm{Ar}^{2+}$ have the same temperature during the arc stage in the spark channel, hence LTE is reached and allows for calculating the temperature with a relatively small uncertainty. The graph shown in figure 4(b) presents the time evolution of the temperature derived in the centre of the gap from the slope of the fitted lines shown in figure 4(a). The temperature (in the temporal range where the signal-to-noise ratio permitted the calculations) has a peak value of $\sim(20000 \pm 480) \mathrm{K}$ and decreases to $\sim(16600 \pm 280) \mathrm{K}$ along with the decrease of the amplitude of the current oscillations.

The emission spectroscopic analysis of arc discharges in argon is investigated in detail for tungsteninert gas (TIG) welding. Recently a thorough study was published on the temperature distribution of TIG arcs in the current range of 100-200 A, where peak temperatures of about 17000-19500 K were reported near the cathode [42]. These values agree fairly well with the temperature obtained here in the 0.5-2.0 $\mu$ s time range in which the peak current is also around 100-200 A.

\subsection{Characterization of the discharge during the afterglow}

In the afterglow stage, i.e. well after the conductive channel had ceased, light emission from the spark gap is still detectable even though no (further) energy is transferred to the spark gap from the capacitor. It is apparent from figure 5(a), that this sustained radiation is produced by excited atomic species. The intensity evolution of an $\mathrm{Ar} I$ and $\mathrm{Cu}$ I spectral lines are shown in figure 5(a). The Ar I emission peaks within the arc stage of the discharge, and it sustains until approx. $50 \mu$ s. The emission 
of copper atoms ceases approximately at the same time as that of the argon, but it reaches its peak intensity considerably later, at around $20 \mu$ s after the breakdown.
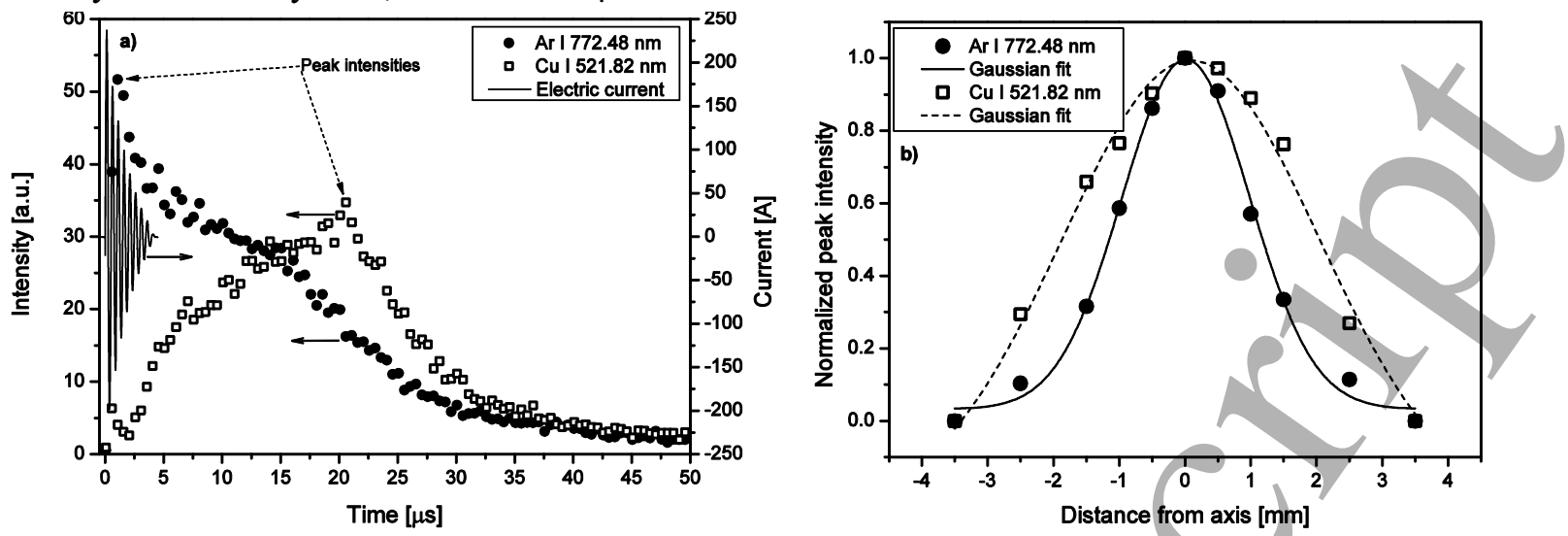

Figure 5. Time evolution of the intensity of some selected atomic spectral lines, emitted from the centre of the gap (a). The spatial variation of the peak intensity of some specific atomic spectral lines perpendicular to the electrodes' common axis, at the centre of the gap (b).

Figure 5(b) shows the spatial distribution of the emission of atomic $\mathrm{Cu}$ and Ar when their respective peak intensity is reached. Unlike ions in the arc stage, the peak intensity of atomic spectral lines shifts towards longer times as a function of the distance from the electrodes' common axis. This suggests that during the afterglow, atomic species are spreading outwards from the centre line of the spark channel, where ions exist in the arc stage and their emission exhibits spatial distributions having a characteristic FWHM of 2.3 and $4.6 \mathrm{~mm}$ for $\mathrm{Ar}$ and $\mathrm{Cu}$, respectively. The broader intensitydistribution of the atomic copper lines are probably due to the much lower excitation energy of $\mathrm{Cu}$, as compared to Ar atoms, therefore excited and emitting $\mathrm{Cu}$ species can exist also in the outer, cooler region of the discharge.

We used the following model to derive the temperature and estimate the composition of the discharge in the afterglow regime. The model is based on the following assumptions: $i$ ) atmospheric pressure prevails in the spark gap, ii) the discharge is only composed of electrons and the neutral and singly charged ionic species of the ambient gas as well as that of the electrode material (here: electrons, $\mathrm{Cu}$, $\mathrm{Cu}^{+}, \mathrm{Ar}, \mathrm{Ar}^{+}$), iii) LTE holds, iv) the net charge of the discharge is zero. These assumptions can be expressed via the following equations:

$$
\begin{gathered}
n_{C u^{+}}+n_{A r^{+}}=n_{e} \\
p=\left(n_{C u^{0}}+n_{A r^{0}}+n_{C u^{+}}+n_{A r^{+}}+n_{e}\right) k_{B} T \\
I_{C u^{0}}=h v_{C u^{0}} A_{C u^{0}} \frac{1}{Z_{C u^{0}}} g_{C u^{0}} \exp \left(\frac{-E_{C u^{0}}}{k_{B} T}\right) n_{C u^{0}}, I_{A r^{0}}=h v_{A r^{0}} A_{A r^{0}} \frac{1}{Z_{A r^{0}}} g_{A r^{0}} \exp \left(\frac{-E_{A r^{0}}}{k_{B} T}\right) n_{A r^{0}} \\
\frac{n_{e} n_{C u^{+}}}{n_{C u^{0}}}=2 \frac{z_{C u^{+}}}{z_{C u^{0}}}\left(\frac{2 \pi m_{e} k_{B} T}{h^{2}}\right)^{\frac{3}{2}} \exp \left(-\frac{W_{C u^{+}}-\Delta W}{k_{B} T}\right), \frac{n_{e^{n} n_{A r^{+}}}}{n_{A r^{0}}}=2 \frac{Z_{A r^{+}}}{Z_{A r^{0}}}\left(\frac{2 \pi m_{e} k_{B} T}{h^{2}}\right)^{\frac{3}{2}} \exp \left(-\frac{W_{A r^{+}}-\Delta W}{k_{B} T}\right)
\end{gathered}
$$

where $n_{C u 0}, n_{A r} 0, n_{C u^{+}}, n_{A r^{+}}$and $n_{e}$ are the concentration of the copper atoms, argon atoms, copper ions, argon ions and electrons, respectively. $p$ is the pressure in the gap, $T$ is the equilibrium plasma temperature, $I_{C u 0}, I_{A r} 0, v_{C u} 0, v_{A r} 0$ are the intensity and the frequency and $g_{C u} 0, g_{A r 0}$ and $E_{C u 0} E_{A r 0}$ are the statistical weight and the energy of the upper state of a specific $\mathrm{Cu}$ I and Ar I spectral line, respectively. $Z_{C u^{+}}, Z_{C u 0}, Z_{A r}, Z_{A r}$ are the partition functions of $\mathrm{Cu}$ and $\mathrm{Ar}$ ions and atoms, respectively, $h$ is the Planck's constant, $k_{B}$ is the Boltzmann constant, $m_{e}$ is the mass of electron, $W_{C u^{+}}$and $W_{A r^{+}}$are the first ionization energy of $\mathrm{Cu}$ and $\mathrm{Ar}$, respectively and $\Delta \mathrm{W}$ is the decrease in the ionization energy according to Griem [40]. (6) expresses the neutrality criterion, (7) is Dalton's law, (8) is the emission 
intensity of an atomic copper and argon line, while (9) is the Saha-Boltzmann equation describing the ratio of the concentration of $\mathrm{Cu}$ and $\mathrm{Ar}$ ions and atoms.

Two of the above given assumptions need to be corroborated. First, one has to assume that the discharge is under atmospheric pressure during the afterglow stage. The breakdown is accompanied by a pressure peak which can be as high as 120 bar in case of a nanosecond spark discharge [43]. This peak is followed by an exponential decrease so the pressure is close to atmospheric pressure again by the end of the arc stage $[43,44]$. Although the exact pressure values might depend on the experimental conditions, considering the rapid decrease it is safe to assume that atmospheric pressure is quickly reached again about $10 \mu$ s after the onset of the breakdown.

The other main assumption of (6)-(9) is the LTE condition during the afterglow. As it was pointed out earlier it is mostly accepted that LTE is reached in a few tens of nanoseconds after the onset of the breakdown in spark discharges. However, it is not trivial how long this equilibrium prevails during the afterglow stage of the discharge. Since the electron concentration is decreasing, the spark is recombining during the afterglow, therefore it is clear that time-averaged LTE-based techniques could not be applied, so temporally resolved measurements are needed. We assume that the discharge evolves through quasi-equilibrium states and LTE conditions hold during the $500 \mathrm{~ns}$ temporal window of the spectral acquisition and (6)-(9) can be applied to characterize the spark in a certain spatial and temporal point. Considerations about the validity of this assumption will be made later in this section (see the last paragraph of this section).

The intensity ratio of atomic copper and argon lines can be calculated from (6)-(9) in a certain temperature and electron concentration range. By comparing the calculated values with experimentally determined intensity ratios the plasma parameters can be deduced. The measured intensity ratios of two $\mathrm{Cu}$-Ar line pairs (Cu I $521.82 \mathrm{~nm} / \mathrm{Ar}$ I $738.40 \mathrm{~nm}$ and $\mathrm{Cu}$ I $515.32 \mathrm{~nm} / \mathrm{Ar}$ I $751.53 \mathrm{~nm}$ ) were used to find solutions of (6)-(9). In order to find the best possible solution Saha-Boltzmann plots were constructed incorporating atomic emission lines of both argon and copper. This requires the modification of (4) by weighting the left side with the concentration of the given species (denoted as $n$ ) calculated from (6)-(9). It is expected that if both Saha and Boltzmann equilibrium conditions are met the points belonging to argon and copper lines lie on the same linear. A further verification of the calculated data was made by simulating the emission spectrum (including the calculation of the Voigt line profiles dependent on the derived plasma parameters) and comparing it to the measured spectrum. By using this model, the discharge parameters were estimated from the emission spectra collected $20.5 \mu$ s after the breakdown, namely at the time when the $\mathrm{Cu}$ I emission is about its maximum. The multi-element Saha-Boltzmann plot obtained from $6 \mathrm{Cu} \mathrm{I}$ and $4 \mathrm{Ar}$ I spectral lines (see Table 1) measured around the centre of the gap is shown in figure 6(a). It can be seen that the points at similar energies overlap and both $\mathrm{Cu}$ and Ar lines nicely fit on the same line indicating the consistency of the results. The error of the derived temperatures comes from the error of the slope of the linear fit to the points of the multi-element Saha-Boltzmann plot and ignores the uncertainty of the constants used for the calculations (see the Methods section). This translates to an absolute temperature error in the range of $130-300 \mathrm{~K}$ for the most cases discussed in the present study. It should be noted, that the concentration values derived from the model are fairly sensitive to the changes in the temperature, so spectral lines having upper energy spread as wide as possible should be incorporated in the SahaBoltzmann plot in order to maximize the accuracy of temperature determination (c.f. the comparison of the accuracy of the Boltzmann and Saha-Boltzmann methods in Section 2.3). The emission spectrum of the three most dominant $\mathrm{Cu}$ I spectral lines simulated at the plasma conditions derived from (6)-(9) is shown in figure 6(b) together with the measured spectrum. The effect of the error of the temperature calculation on the simulated spectrum is indicated by a grey zone around the solid, black curve. 

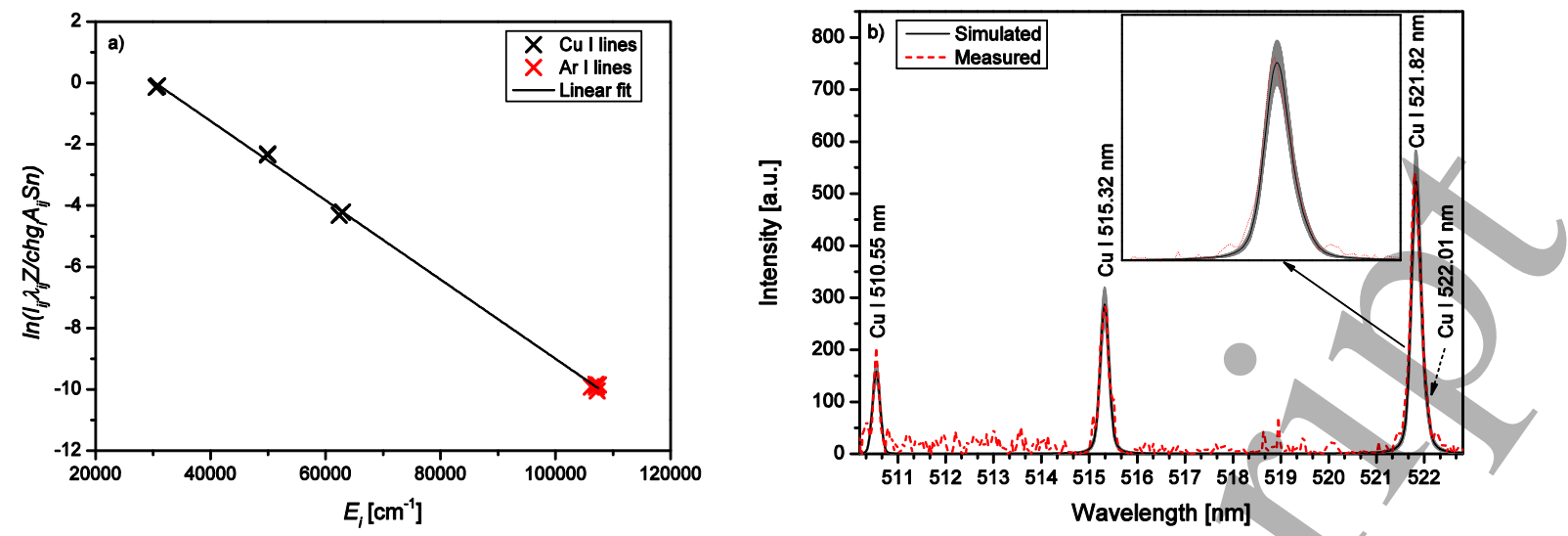

Figure 6. Multi-element Saha-Boltzmann plot of atomic Ar and $\mathrm{Cu}$ lines (a) and the spectrum of the three most dominant $\mathrm{Cu}$ I lines measured (dashed curves) and simulated (solid curves) around the centre of the gap at $20.5 \mu \mathrm{s}$ after the breakdown. The error is shown as a grey zone around the simulated spectrum. The $521.82 \mathrm{~nm}$ peak is shown magnified in the inset.

It can be seen from figure 6(b) that the simulated spectrum fits the measured one neatly, which suggests that the initially assumed LTE condition is met and our model describes the spark in the SDG during the afterglow stage fairly well. The calculated parameters are summarized in table 2 . It can be seen that the temperature is about $(10820 \pm 130) \mathrm{K}$. The electron concentration is ca. $\left(3.59 \times 10^{16} \pm 0.39 \times 10^{16}\right) \mathrm{cm}^{-3}$ which is about one order of magnitude smaller than the characteristic value at the end of the arc stage (cf. figure 3 ).

Table 2. Calculated parameter values for the centre of the gap $20.5 \mu$ s after the breakdown. The error of the concentrations was derived from the error of the temperature determined from the SahaBoltzmann plot.

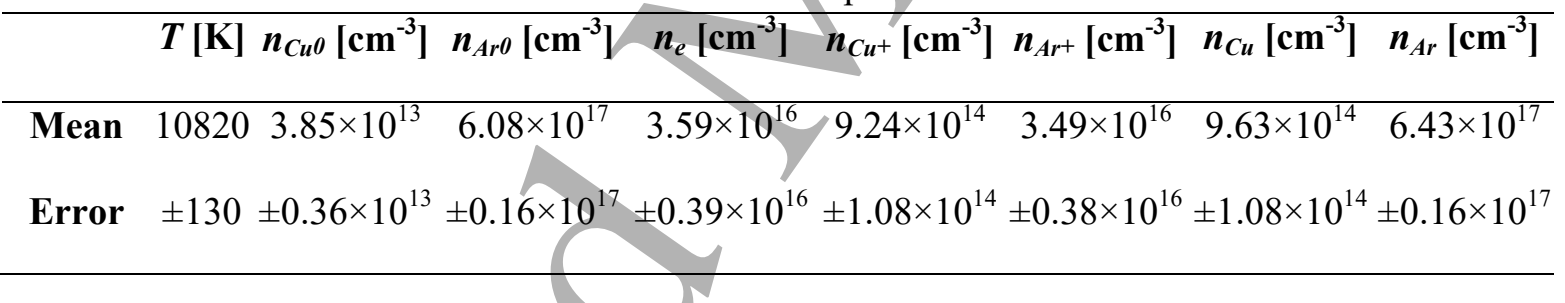

Fast imaging was used in order to acquire data on the shape of the discharge in the SDG during the afterglow. Generally, the cylindrical shape of the discharge which is characteristic to the arc stage, becomes more diffuse after the current stops flowing between the electrodes [18]. The image of the spark gap $20.5 \mu$ s after the breakdown is shown in figure 7 .

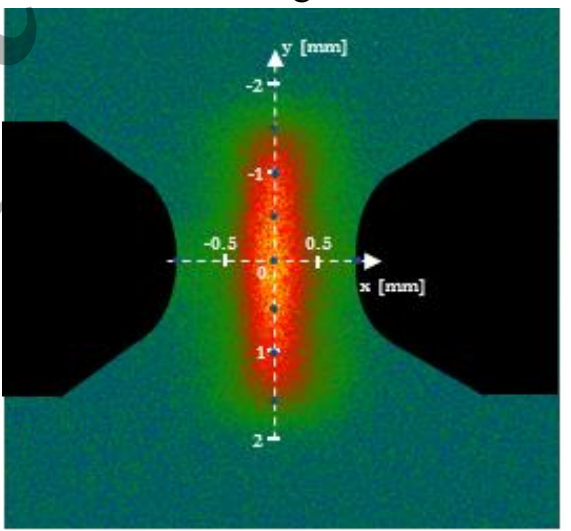

Figure 7. Image of the electrode gap taken with $100 \mathrm{~ns}$ exposure time at $20.5 \mu$ s after the breakdown. The points where emission spectra were taken are indicated by the blue dots along the axes. 
The image of the spark, i.e. a 2D projection, shows the presence of an emitting, more-or-less rectangular area having dimensions of $\sim 3 \mathrm{~mm}$ in height and $\sim 1 \mathrm{~mm}$ in width. Please note, that the emission collected by the imaging iCCD is spectrally integrated, thus the image cannot be directly connected to the distribution of a specific species. It suggests that the overall emission of the excited species is approximately homogenous in the central core. However, by evaluating the spectrally resolved emission taken at different spatial points (indicated by blue dots in figure 7) the distribution of the spark parameters, namely temperature and electron concentration, can be derived from the semiempirical model based on (6)-(9). The calculated temperatures and electron concentrations and their variation along and perpendicular to the electrode axis are shown in figure 8.
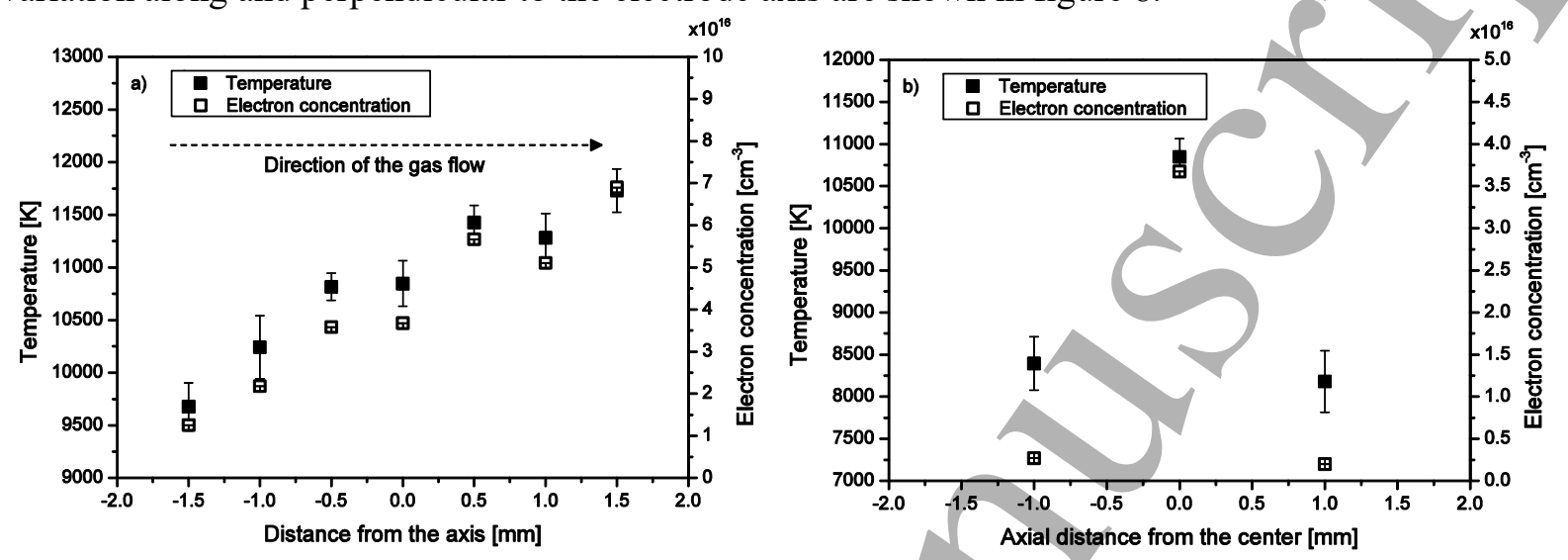

Figure 8. Spatial variation of temperature and electron concentration perpendicular to (a) and along (b) the electrodes' common axis $20.5 \mu$ s after the breakdown.

As can be seen in figure 8(a) the temperature and electron concentration exhibit an increasing trend perpendicular to the electrodes' common axis in the direction of the gas flow. This result suggests that the $5 \mathrm{l} / \mathrm{min}$ gas flow employed in our SDG has an effect on the spark parameters resulting in higher temperature and larger electron concentration downstream of the flow. Contrary to this, the variation of the temperature and electron concentration along the electrodes' common axis is symmetric, as shown in figure $8(b)$.

The distribution of the $\mathrm{Ar}$ and $\mathrm{Cu}$ species in the gap was also investigated by employing our model to estimate the concentration of each species at different spatial positions.
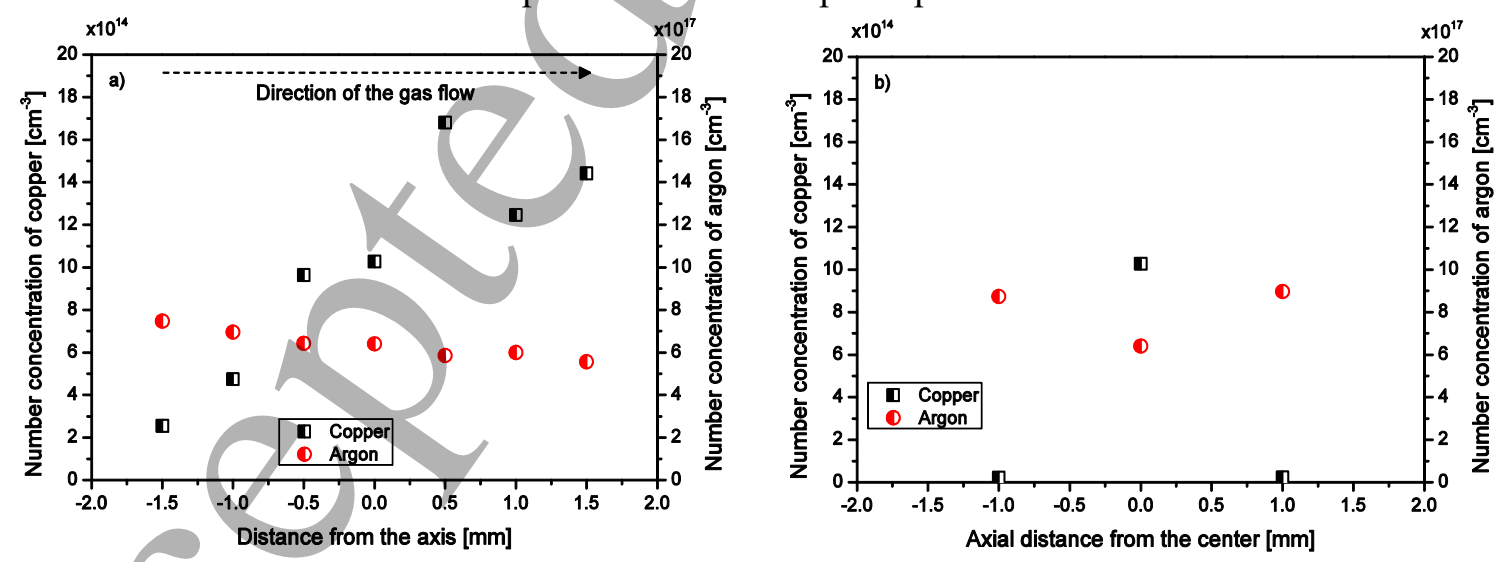

Figure 9. Spatial distribution of total number concentration of copper and argon species (i.e. the sum of the atomic and ionic species) perpendicular to (a) and along (b) the electrodes' common axis, $20.5 \mu$ s after the breakdown.

As can be seen in figure 9(a) the concentration of argon (i.e. the sum of the atomic and ionic species) is about 2-3 orders of magnitude higher than the average number concentration of copper. The number concentration of copper increases, while the argon concentration slightly decreases in the direction of 
the gas flow. The increasing amount of $\mathrm{Cu}$ is probably due to the fact that the majority of copper is in ionic form whose concentration increases with increasing temperature.

In the axial direction, the argon concentration exhibits slight and symmetrical changes, but the total concentration of copper species varies significantly in the $-0.5 \mathrm{~mm}-+0.5 \mathrm{~mm}$ axial range. It is, by a factor of about 50, smaller close to the electrode tips as compared to the value obtained in the middle of the gap. The results are summarized in figure 9(b). The two distributions shown in figure 9 suggest that at $20.5 \mu \mathrm{s}$, the copper plume is concentrated in the middle of the gap and exhibits an increasing concentration along the direction of the carrier gas flow.

The temporally resolved data together with the employed model also allow for estimating the rate of change of temperature and electron concentration during the afterglow. By evaluating the data in the centre of the gap $\frac{d T}{d t} \approx-3.4 \times 10^{8} \frac{K}{\mathrm{~s}}$ and $\frac{d n_{e}}{d t} \approx-1.4 \times 10^{22} \frac{\mathrm{cm}^{-3}}{\mathrm{~s}}$ are obtained. From this it follows that the temperature and the electron concentration changes by $\sim 170 \mathrm{~K}$ and $\sim 7 \times 10^{15} \mathrm{~cm}^{-3}$, respectively during the $500 \mathrm{~ns}$ temporal window of the spectral acquisition, which corresponds to a $\sim 1.5 \%$ and $\sim 20 \%$ change of the temperature and electron concentration, respectively. The electron concentration is frequently used to estimate whether LTE exists in a spark plasma or not by comparing it to a critical value calculated from the so-called Griem criterion [32]. Although one cannot rely solely on the electron concentration, this criterion could provide a rough estimate of the temporal window in which LTE holds in the afterglow. From the values reported earlier we obtained that LTE does hold in our system until around $24 \mu$ s after the breakdown.

\subsection{Implications of the discharge characteristics on the NP generation}

It was shown in the previous chapters that the arc stage of an SDG spark is characterized by high temperatures and high electron concentrations, caused by the high electric current, which in turn is responsible for the erosion and vaporization of the electrode material. In the afterglow stage, the vapour cloud reaches more moderate temperatures and emits light until it is cold enough that particle formation mechanisms can commence, Therefore, the afterglow can be considered to be the real starting point of NP generation. Hence, spectroscopic investigation of the afterglow can be a valuable tool for providing estimates of species concentration and temperature data for establishing realistic initial conditions for nucleation and growth models.

A key parameter in all existing particle models, which aim to predict the size distribution of NPs generated by sources such as the spark discharge, is the number of atoms produced by a single spark which is considered to determine the final particle size $[6,15]$. The number of the produced atoms is related to the mass of the electrode material eroded and atomized by a single spark. This quantity is usually derived by a simple evaporation model based on the energy balance, often referred to as the Jones model [45]. The Jones model can acceptably predict the ablated mass for several electrode materials, but only via an empirical factor, which has to be determined by gravimetric analysis. A serious shortcoming of the NP evolution calculations which rely on this model is the implicit assumption that all the material eroded by a spark is thermally vaporized and is fed into the NP formation process. However, there are experimental evidences that this is not necessarily the case. Most notably, micrometre sized electrode fragments, so-called "splashing particles", are also removed from the electrodes [5]. These relatively large particles contribute substantially to the measured massloss of the electrodes, but are not NPs and are not formed via the conventional thermal, i.e. evaporation-condensation route of particles. This could result in the overestimation of the initial number concentration of the atomized electrode material if the Jones model is mechanistically employed.

At the same time, the model described above does not rely on the measurement of the ablated mass per spark, but is instead based on the direct measurement of the emission of the spark in the afterglow 
regime, i.e. before the initiation of particle formation. Hence it is able to provide more realistic information about the boundary conditions of the NP formation process, thus can contribute to the better prediction of NP properties and ultimately to a better understanding of the NP formation mechanisms.

\section{Conclusions}

A spark discharge, run in argon and used for copper NP generation, was characterized by means of spatially and temporally resolved optical emission spectroscopy (OES) parallel with fast imaging. The acquired spectral and imaging data were used to describe the spatial and temporal evolution of the species, and the plasma conditions that exist in the spark gap, especially in its arc and afterglow stages. We found that in the arc stage a spatially concise conducting channel is formed by $\sim 500 \mathrm{~ns}$ after the onset of the breakdown, that has a diameter of about $0.7 \mathrm{~mm}$ and is composed of mainly ionic and atomic species of the ambient gas and maintains the current between the electrodes during the arc phase, i.e. approx. $4.5 \mu$ s. Stark broadening and Saha-Boltzmann plot of emission lines of argon atoms and ions were used to describe the electron concentration and temperature in the arc stage. It was found that the temperature varies between $\sim(20000 \pm 480) \mathrm{K}$ and $\sim(16600 \pm 280) \mathrm{K}$ while the electron concentration decreases from $\sim 7 \times 10^{17} \mathrm{~cm}^{-3}$ to around $2 \times 10^{17} \mathrm{~cm}^{-3}$ as the current ceases in an oscillatory fashion. These temperature and electron concentration values are in line with the assumption that LTE prevails in the spark channel.

The arc stage is followed by an about $45 \mu$ s long afterglow, in which excited atomic species dominate the emission in the spark gap. The light emitting atoms are distributed in a more extended volume, as compared to the spark channel, and the plume reaches a maximum diameter of about $4.6 \mathrm{~mm}$ (as defined by the FWHM of the $\mathrm{Cu}$ distribution) by the end of the afterglow stage. We described this stage by an equilibrium model. The temperature and electron concentration in the spark gap at $20.5 \mu \mathrm{s}$ after the breakdown was found to be increasing perpendicular to the electrodes' common axis in the direction of the carrier gas flow, having a value of $(10820 \neq 130) \mathrm{K}$ and ca. $\left(3.59 \times 10^{16} \pm 0.39 \times 10^{16}\right) \mathrm{cm}^{-3}$, respectively in the centre of the gap. The concentration of $\mathrm{Cu}$ species - which is fairly sensitive to the changes in the temperature - was also estimated in the electrode gap at this time and a value of $\left(9.63 \times 10^{14} \pm 1.08 \times 10^{14}\right) \mathrm{cm}^{-3}$ was obtained in the centre of the gap. The $\mathrm{Cu}$ concentration was found to be by a factor of 50 lower in the vicinity of the electrodes' tips. Our results suggest that the $\mathrm{Cu}$ vapour is concentrated in the centre of the gap, and its concentration is increasing in the direction of the gas flow.

We conclude that temporally and spatially resolved OES together with a simple equilibrium model are efficient tools to characterize the spark discharge plasma that typically exists in SDGs. Furthermore, it was also shown that our OES data-based calculations are able to approximate the electrode material concentration which could be used to complement the data obtained by gravimetry-based methods. Our results therefore can be used to fine-tune the NP evolution models which aim to describe the vivid physical and chemical processes taking place in an SDG.

\section{Acknowledgments}

The research leading to these results has received funding from the European Union's Seventh Framework Program under Grant Agreement No. 280765 (BUONAPART-E).

\section{References}

[1] Schwyn, T. V., Garwin, E., \& Schmidt-Ott, A. (1988). Aerosol generation by spark discharge. Journal of Aerosol Science, 19, 639-642.

[2] Byeon, J. H., Park, J. H., \& Hwang, J. (2008). Spark generation of monometallic and bimetallic aerosol nanoparticles. Journal of Aerosol Science, 39, 888-896. 
[3] Meuller, B. O., Messing, M. E., Engberg, D. L. J., Jansson, A. M., Johansson, L. I. M., Norlén, S. M., Tureson, N., \& Deppert, K. (2012). Review of spark discharge generators for production of nanoparticle aerosols. Aerosol Science and Technology, 46, 1256-1270.

[4] Pfeiffer, T. V., Feng, J., \& Schmidt-Ott, A. (2014). New developments in spark production of nanoparticles. Advanced Powder Technology, 25, 56-70.

[5] Tabrizi, N. S., Ullmann, M., Vons, V. A., Lafont, U., \& Schmidt-Ott, A. (2009). Journal of Nanoparticle Research, 11, 315-332.

[6] Feng, J., Biskos, G., \& Schmidt-Ott, A. (2015). Toward industrial scale synthesis of ultrapure singlet nanoparticles with controllable sizes in a continuous gas-phase process. Scientific Reports, $\mathbf{5}, 15788$.

[7] http://www.buonapart-e.eu/

[8] Watters, R. L. Jr., DeVoe, J. R., Shen, F. H., Small, J. A., \& Marinenko, R. B. (1989). Characteristics of Aerosols Produced by the Spark Discharge. Analytical Chemistry, 61, 18261833.

[9] Nilsson, P. T., Eriksson, A. C., Ludvigsson, L., Messing, M. E., Nordin, E. Z., Gudmundsson, A. Meuller, B. O., Deppert, K., Fortner, E. C., Onasch, T. B., Pagels, J. H. (2015). In-situ characterization of metal nanoparticles and their organic coatings using laser-vaporization aerosol mass spectrometry. Nano Research, 8, 3780.

[10] Ludvigsson, L., Meuller, B. O., \& Messing, M. E. (2015). Investigations of initial particle stages during spark discharge. Journal of Physics D, 48, 314012.

[11] Hontañon, E., Palomares, J. M., Stein, M., Guo, X., Engeln, R., Nirschl, H., \& Kruis, F. E. (2013). The transition from spark to arc discharge and its implications with respect to nanoparticle production. Journal of Nanoparticle Research, 15, 1957.

[12] Byeon, J. H. \& Kim, J-W. (2010). Morphology and Structure of Aerosol Carbon-Encapsulated Metal Nanoparticles from Various Ambient Metal-Carbon Spark Discharges. Applied Materials \& Interfaces, 2(4), 947-951.

[13] Boddu, S. R., Gutti, V. R., Ghosh, T. K., Tompson, R. V., Loyalka, S. K. (2011). Gold, silver, and palladium nanoparticle/nano-agglomerate, generation, collection, and characterization. Journal of Nanoparticle Research, 13, 6591-6601.

[14] Borra, J. P. (2006). Nucleation and aerosol processing in atmospheric pressure electrical discharges: powders production, coatings and filtration. Journal of Physics D. 39, R19-R54.

[15] Feng, J., Huang, L., Ludvigsson, L., Messing, M. E., Maisser, A., Biskos, G., \& Schmidt-Ott, A. (2016). General Approach to the Evolution of Singlet Nanoparticles from a Rapidly Quenched Point Source. Journal of Physical Chemistry C, 120, 621-630.

[16] Itina, T. E., Voloshko, A. (2013). Nanoparticle formation by laser ablation in air and by spark discharges at atmospheric pressure. Applied Physics B, 113, 473.

[17] Voloshko, A., Colombier, J-P., Itina, T. E. (2014). Comparison of laser ablation with spark discharge technique used for nanoparticle production. Applied Surface Science, 336, 143-149.

[18] Palomares, J. M., Kohut, A., Galbács, G., Engeln, R., Geretovszky Zs. (2015). A time-resolved imaging and electrical study on the operation of a high current atmospheric pressure spark discharge. Journal of Applied Physics, 118, 233305.

[19] Pai, D. Z., Lacoste, D. A., \& Laux, C. O. (2010). Nanosecond repetitively pulsed discharges in air at atmospheric pressure - the spark regime. Plasma Sources Science and Technology, 19, 065015.

[20] Janda, M., Machala, Z., Niklová, A., \& Martišovitš, V. (2012). The streamer-to-spark transition in a transient spark: a dc-driven nanosecond-pulsed discharge in atmospheric air. Plasma Sources Science and Technology, 21, 045006.

[21] Bazelyan, E. M. \& Raizer, Y. P. (1997). Spark Discharge. Boca Raton: CRC Press. 
[22] Aleksandrov, N. L. \& Bazelyan, E. M. (1999). Ionization processes in spark discharge plasmas. Plasma Sources Science and Technology, 8, 285-294.

[23] Walters, J. P. (1969). Historical advances in spark emission spectroscopy. Applied Spectroscopy, 23, 317-331.

[24] Bardócz, Á. \& Varsanyi, F. (1956). Spectrochemical analysis with time-resolved spark spectra. Nature, 177, 222-223.

[25] Scheeline, A. \& Coleman, D. M. (1987). Direct Solids Elemental Analysis: Pulsed Plasma Sources. Analytical Chemistry, 59, 1185-1196.

[26] Boumans, P. W. J. M. (1972). Excitation of spectra. In E. L. Grove (Ed), Analytical Emission Spectroscopy. New York: Marcel Dekker.

[27] Walters, J. P. \& Malmstadt, H. V. (1965). Emission Characteristics and Sensitivity in a HighVoltage Spark Discharge. Analytical Chemistry, 37, 1484-1503.

[28] Raizer, Y. P. (1991). Gas discharge physics. Berlin Heidelberg: Springer-Verlag.

[29] Scheeline, A., Norris, J. A., Travis, J. C., DeVoe, J. R., Walters, J.P. (1981). Particulates formed by a stabilized high voltage spark discharge. Spectrochimica Acta B, 36, 373-383.

[30] Bardócz, Á. \& Vörös, T. (1965). The determination of the plasma temperature with the aid of Stark broadening of spectrum lines with an application to transient/discharges. Journal of Quantitative Spectroscopy and Radiative Transfer, 6, 351-356.

[31] Diermeier, R. \& Krempl, H. (1970). Zeitaufgelöste Spektren im Funkenplasma. Spectrochimica Acta, 25B, 69-81.

[32] Bye, C. A. \& Scheeline, A. (1993). Saha-Boltzmann Statistics for Determination of Electron Temperature and Density in Spark Discharges Using an Echelle/CCD System. Applied Spectroscopy, 47, 2022-2030.

[33] Bye, C. A. \& Scheeline, A. (1993). Stark electron density mapping in the high voltage spark discharge. Spectrochimica Acta, 48B, 1593-1605.

[34] Bye, C. A. \& Scheeline, A. (1995). Electron density profiles in single spark discharges. Journal of Quantitative Spectroscopy and Radiative Transfer, 53, 75-93.

[35] Blitzer, L \& Cady, W. M. (1951). Excitation Temperature in Time-Resolved Spectra of Single Condensed Spark Discharges. Journal of the Optical Society of America, 41, 440-445

[36] Aragón, C. \& Aguilera, J. A. (2008). Characterization of laser induced plasmas by optical emission spectroscopy: A review of experiments and methods. Spectrochimica Acta Part B, 63, 893-916.

[37] http://physics.nist.gov/PhysRefData/ASD/lines form.html

[38] Konjevic, N. (1999). Plasma broadening and shifting of non-hydrogenic spectral lines: present status and applications. Physics Reports, 316, 339-401.

[39] Aparicio, J. A., Gigosos, M. A., Gonzalez, V. R., Pérez C., de la Rosa, M. I., \& Mar, S. (1998). Measurement of Stark broadening and shift of singly ionized Ar lines .Journal of Physics B, 31, 1029-1048.

[40] Griem, H. R. (1962). High-Density Corrections in Plasma Spectroscopy, Physical Review, 128, 997.

[41] Mandelstam, S. (1959). Excitation of the spectrum in a spark discharge. Spectrochimica Acta, 255-271.

[42] Bachmann, B., Kozakov, R., Gött, G., Ekkert, K., Bachmann, J-P., Marques, J-L., Schöpp, H., Uhrlandt, D. and Schein, J. (2013). High-speed three-dimensional plasma temperature determination of axially symmetric free-burning arcs, Journal of Physics D, 46, 125203.

[43] Reinmann, R. and Akram, M. (1997). Temporal investigation of a fast spark discharge in chemically inert gases. Journal of Physics D, 30, 1125. 
[44] Voloshko, A. (2016). Formation of nanoparticles by means of spark discharge at atmospheric pressure. PhD thesis, University Jean Monnet.

[45] Jones, F. L. (1950). Electrode Erosion by Spark Discharges. British Journal of Applied Physics, 1, 60-65. 